\title{
«Ein Organ ist ein Geschenk»
}

\section{Daniel Lüthi}

Freier Journalist, Fotograf, Medientrainer, Bern

\author{
Um Transplantationen und Menschenrechte ging es an diesem internationalen \\ Expertenforum in Bern, um Medizin und Moral, Recht und Ethik. Vor allem aber \\ ging es um China. Dort sei bei Zehntausenden von transplantierten Organen die \\ Herkunft unbekannt, schickte die Gesellschaft für Menschenrechte voraus, die den \\ Anlass organisiert hatte. Politische Gefangene würden ihrer Organe beraubt und \\ dadurch umgebracht.
}

Die 43-jährige Liu Wei aus der chinesischen Provinz Liaoning kam 2004 nach Deutschland, um dort zu studieren. Als aktives Mitglied der Bewegung Falun Gong, die in China verboten ist, sei sie zwischen 2001 und 2003 in verschiedenen chinesischen Gefängnissen und Arbeitslagern eingesperrt gewesen, erzählte sie in Bern. Zum Teil unter Tränen berichtete sie von schweren körperlichen und seelischen Misshandlungen, die sie erlebt habe. Fünfmal sei sie in ihrer Gefangenschaft zusammen mit anderen Falun-Gong-Anhängern - von Kopf bis Fuss medizinisch untersucht worden, ohne dass sie je über die Resultate unterrichtet worden wäre. «Es war klar», sagte sie, «das waren Vorbereitungen für einen Organ-Raub.»

Auf die Frage der SÄZ, ob das eine Vermutung sei oder ob sie Beweise habe, antworte Liu Wei: «Der Polizist sagte nichts von Organ-Entnahmen.» Das Muster wiederhole sich immer und immer wieder, die Indizien für

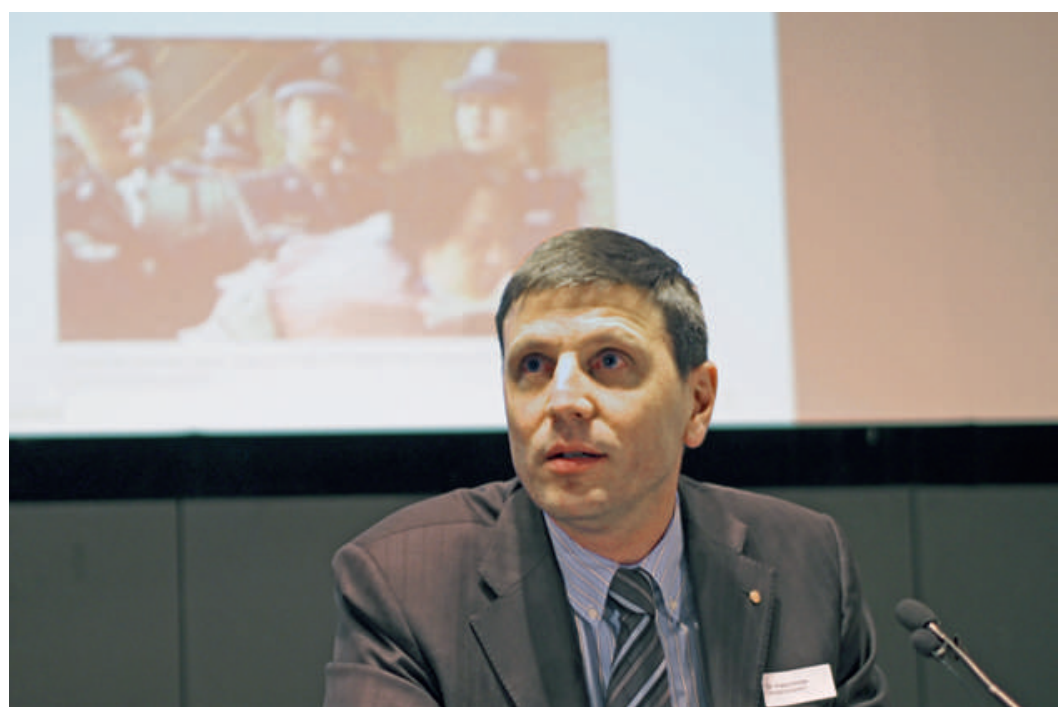

Franz Immer, Herzchirurg und CEO Swisstransplant.

\section{"Un organe est un don"}

Le premier forum international d'experts sur la transplantation et les droits humains qui s'est tenu le 16 avril à Berne, s'est penché sur les questions de la médecine et de la morale ainsi que du droit et de l'éthique, en se concentrant principalement sur la Chine. Selon la société pour les droits humains qui a organisé la manifestation, des dizaines de milliers d'organes transplantés en Chine seraient d'origine inconnue, et des prisonniers politiques seraient tués pour leurs organes.

einen massiven kriminellen Handel mit Organen in China seien erdrückend, ergänzten Menschenrechtsvertreter.

\section{Nicht nur in China}

Franz Immer, Herzchirurg und Geschäftsführer von Swisstransplant, bestätigte die Wahrnehmung, dass es viele und starke Indizien gibt, der direkte Bezug zwischen den systematischen Blut- und Gesundheitstests und späterem Organraub jedoch nicht hundertprozentig festzumachen ist (vgl. auch untenstehendes Interview). Weiter sagte er: «Dass Hingerichteten Organe entnommen werden, geschieht nicht nur in China. In den USA, im Staat Utah, ist ein Gesetz angenommen worden, das dies erlaubt. Organ- und Gewebehandel geschehen oft auf Kosten armer Menschen und deshalb vor allem in Entwicklungsländern.» Gegen jegliche Art von Handel mit Gewebe und Organen sei entschieden vorzugehen. "Zentral sind Respekt und Würde eines Verstorbenen und der freie Wille. Eine Organ- oder Gewebespende ist ein Geschenk an einen Menschen, der auf der Warteliste steht.» 


\section{Und die Schweiz?}

"Ein Organ ist ein Geschenk», sagte an der Tagung auch Prof. Gerhard Dannecker, Direktor des Instituts für Strafrecht und Strafprozessrecht an der Universität Heidelberg. Organhandel sei Teil der internationalen organisierten Kriminalität wie zum Beispiel auch der Drogenhandel. Bei den Medizinern sei dieses Unrechtsbewusstsein noch zu wenig vorhanden. «Den Patienten helfen: ja», sagte er, «aber nur auf legale Weise.» Die Not des einen dürfe nicht einen anderen in Not bringen. Hierzulande bestehe diese Gefahr allerdings kaum, das Schweizer Strafrecht garantiere genügend Schutz: «In der Schweiz leben Sie im Land der Glückseligen.»

David Kilgour und David Matas aus Kanada, Co-Autoren des Buches Blutige Ernte, sagten in ihren ausufernden und zum Teil ziemlich verwirrenden Beiträgen inhaltlich praktisch dasselbe wie ihre Vorredner.

Die Fragerunde förderte dann noch zwei andere Aspekte zutage: Patientenvertreterin und Nationalrätin Margrit Kessler erwähnte als dominierendes Element die massiven wirtschaftlichen Interessen, die Teil dieser Thematik sind, symbolisiert beispielsweise durch das Freihandelsabkommen zwischen der Schweiz und China. Diese Interessen würden es verunmöglichen,

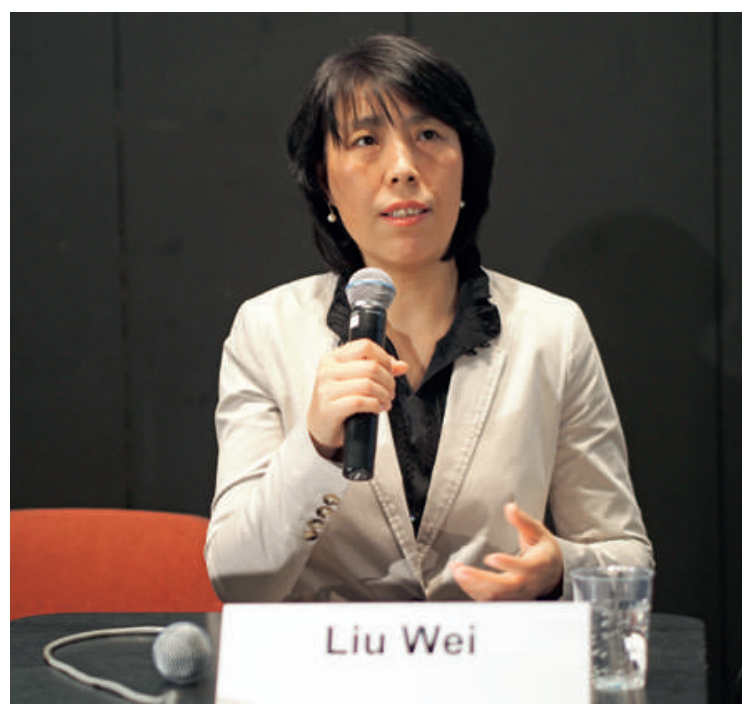

Liu Wei, Folteropfer aus China.

gegen die Verletzung von Menschenrechten in China mit der nötigen Härte zu opponieren.

Und Danielle Costelli, Vertreterin von Amnesty International, erwähnte Pharmafirmen, die Medikamente gegen die Abstossung von transplantierten Organen produzieren. Für ihre Tests bräuchten diese Firmen Organe, und diese würden sie auch aus illegalen Quellen beziehen - beispielsweise von Hingerichteten in China.

\title{
Transparenz und klare Regeln
}

\author{
Interview: Daniel Lüthi
}

\section{Es gibt offensichtlich eine Dringlichkeit, beim Thema "Transplantationen und Menschenrechte» den Fokus auf China zu richten. Bitte führen Sie kurz und möglichst konkret aus, warum.}

Franz Immer: Es gibt in China viel Rauch, so dass man annehmen muss, dass dort auch ein Feuer ist. Sicher ist, dass chinesische Spitäler Organtransplantationen auf ihren Internetseiten angeboten haben, dass FalunGong-Anhänger systematisch verfolgt und inhaftiert wurden, und dass Organe von Exekutierten zur Organspende verwendet wurden, wie dies auch offizielle Quellen bestätigen.

\section{Haben Sie Beweise?}

Klare Evidenzen, dass die Exekutionen in China vor dem Hintergrund der Organspende vollzogen wurden, habe ich keine.
Haben Sie Kenntnis davon, dass auch die Schweiz von Organhandel betroffen ist? Dass hier Menschen gegen ihren Willen Organe entnommen oder anderen Menschen illegal erworbene Organe eingepflanzt werden? Oder können Sie dies ausschliessen? In der Schweiz kann eine illegale Organentnahme und/ oder eine illegale Transplantation von Organen verstorbener Spender ausgeschlossen werden. Das System ist sehr eng kontrolliert - es bestehen Meldepflichten und der Spenderprozess, wie auch die Zuteilung von Organen, sind klar geregelt.

\section{Sind Sie als Herzchirurg schon mal konkret mit dem Thema Organhandel konfrontiert worden?} Ja. Anfang 2000 nahm ich in Peking an einem herzchirurgischen Kongress teil. Damals wurde ich auch eingeladen, ein herzchirurgisches Zentrum zu besuchen. 


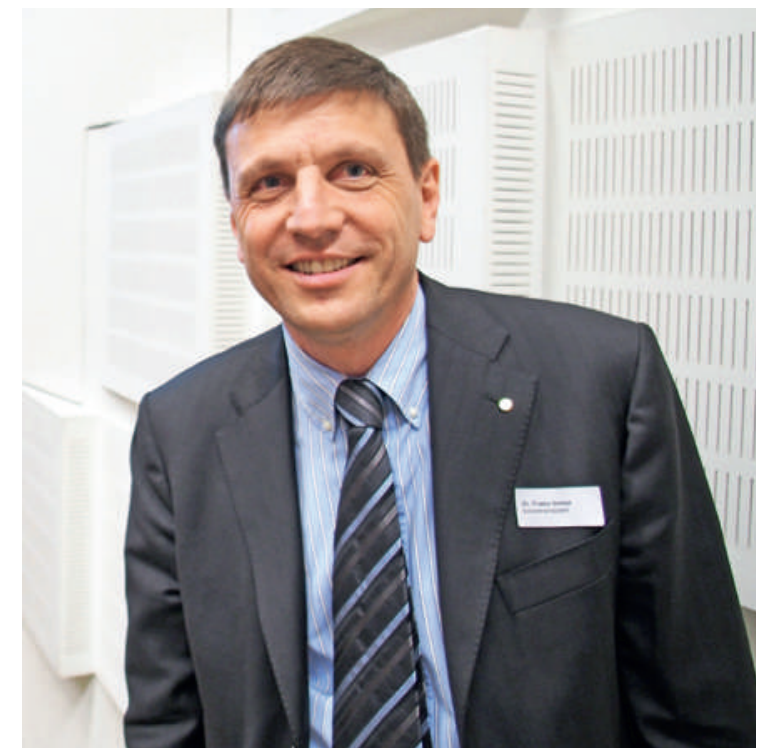

Franz Immer

Mein Interesse war gross, da dort die Zahl der Eingriffe deutlich höher liegt als die Gesamtzahl der Eingriffe schweizweit. Es wurde mir ein Datum vorgeschlagen und als Eingriff wollte man mir eine Herztransplantation zeigen. Weil ich weiss, dass Organspenden und somit Transplantationen nicht planbar sind, habe ich damals diesen Besuch abgelehnt. Ein israelischer Kollege aber berichtete, dass einer seiner Patienten ihm mitgeteilt habe, dass er ein Datum habe für eine Herztransplantation in China. Auch dies überrascht aus obigem Grund und zeigt auf, dass dort ausländische Patienten gegen Entgelt Organe kaufen können.

\section{Bedeutet Ihr Kampf gegen den Organhandel gleich-} zeitig auch einen verstärkten Einsatz für legale, freiwillige Organspenden?

Absolut. Die Illegalität kann nur ein Ende finden, wenn wir uns selber als Menschen Gedanken machen, ob man Organe und Gewebe spenden würde - und im Gegenzug sich vielleicht auch überlegt, ob man eine Organ- oder Gewebespende annehmen würde. Kann man die zweite Frage mit "Ja» beantworten, so ist die Organ- und Gewebespende eigentlich eine Selbstverständlichkeit.

\section{Was sind Ihre Hauptargumente für eine Organ- spende?}

Menschen in Not - im Angesicht des Todes oder in Anbetracht langjähriger Dialysebehandlungen - suchen nach Lösungen. Dies öffnet die Türe zu kriminellen Machenschaften. Hier kann nur ein legales, ethisch korrektes und transparentes Spenderwesen und eine klare Regelung über die Organzuteilung Abhilfe schaffen.

Der Entscheid jedes Einzelnen zählt und muss den nächsten Angehörigen kommuniziert werden. Organspende ist bis ins hohe Alter möglich und es gibt kaum medizinische Ausschlussgründe. Es kann uns somit fast alle betreffen, Spender zu werden. Oder noch viel wahrscheinlicher: auf der Organwarteliste zu sein und selber auf das Geschenk einer Organspende zu hoffen.

\section{Und wenn es in der Schweiz kaum Hoffnung auf ein Organ gibt?}

Der hohe medizinische Standard hierzulande hält die Schweizer Patienten davon ab, Alternativen in Ländern wie China zu suchen. Es ist wichtig, dass die Schweiz die Konvention von Istanbul ratifiziert, um auch extraterritoriale Machenschaften auf dem Gebiet des Organ- und Gewebehandels sanktionieren zu können.

Wie viele Menschen sterben in der Schweiz, weil sie nicht rechtzeitig ein Organ erhalten, das ihnen das Überleben mit grosser Wahrscheinlichkeit gesichert

\section{hätte?}

Wir gehen in der Schweiz von rund 2 Todesfällen pro Woche aus.

\section{Bei welchen Gelegenheiten thematisieren Sie die} Problematik "Organspende und Organhandel» in Ihrem medizinischen Umfeld, in Ihrem beruflichen Alltag als Arzt?

Bei meinen Präsentationen ist die Zusammenarbeit mit anderen nationalen Organisationen, allen voran der Agence de la biomédecine in Frankreich, immer Bestandteil. Ich erwähne dort am Rande jeweils auch den illegalen Organhandel, indem ich aufzeige, wie in der Schweiz die Kontrolle ausgeübt wird.

In der Diskussion mit politischen Instanzen und den Versicherern weise ich immer wieder darauf hin, dass nur eine gute, offene und transparente Spenderarbeit, die auch finanzielle und personelle Ressourcen benötigt, den illegalen Handel unterbinden kann. Die Schweizer Bevölkerung ist über die Sprach- und Konfessionsgrenzen hinweg in einer überwiegenden Mehrheit bereit zur Organ- und Gewebspende. Bei den Jugendlichen wissen wir heute, dass über $85 \%$ eine Organspende bejahen. 